\title{
Psoriatic Arthritis and Psoriasis Projects in Italy: A Report from the GRAPPA 2011 Annual Meeting
}

\author{
ALBERTO CAULI, ENNIO LUBRANO, RAFFAELE SCARPA, and IGNAZIO OLIVIERI
}

\begin{abstract}
Recent evidence that psoriatic arthritis (PsA) is more frequent and more severe than previously thought has led to several studies on genetic susceptibility, pathogenesis, clinical spectrum, diagnostic tools, and treatment of PsA. Concomitant with these studies has been the development of appropriate classification/diagnostic criteria and disease-specific outcome measures for PsA. Many Italian rheumatologists have taken part in these efforts by conducting collaborative multicenter research projects, which were described at the annual meeting of the Group for Research and Assessment of Psoriasis and Psoriatic Arthritis (GRAPPA; Naples, Italy, July 2011). Highlights of these Italian contributions included performance of CASPAR (ClASsification for Psoriatic ARthritis) criteria in early PsA; radiological assessments of axial PsA; development and validation of the PASRI (PsA Spondylitis Radiology Index in patients with established PsA); and the effect of etanercept on axial manifestations of established PsA. (J Rheumatol 2012;39:2201-3; doi:10.3899/ jrheum.120824)
\end{abstract}

Key Indexing Terms:

PSORIATIC ARTHRITIS $\quad$ EARLY DISEASE TREATMENT $\quad$ X-RAY ASSESSMENT

Although psoriatic arthritis (PsA) was considered for many years a mild form of arthritis, recent and consistent evidence has shown that PsA is more frequent and more severe than previously thought. Our knowledge of the genetic susceptibility, pathogenesis, clinical spectrum, diagnostic tools, and treatment of PsA, including the development of anti-tumor necrosis factor (TNF) agents, has advanced interest in PsA as a clinical entity. This interest includes development of appropriate classification/diagnostic criteria and disease-specific outcome measures for PsA, which were previously assessed with tools borrowed from those developed for rheumatoid arthritis (RA) or ankylosing spondylitis (AS).

Many Italian rheumatologists have taken part in these efforts, contributing to the advances in PsA knowledge by proposing and conducting collaborative multicenter research projects. The Group for Research and Assessment of Psoriasis and Psoriatic Arthritis (GRAPPA) recognized these efforts by including at the GRAPPA Annual Meeting

From the Department of Medical Science, Policlinico di Monserrato, University of Cagliari, Monserrato; Rheumatology Unit, Department of Health Sciences, University of Molise, Campobasso; Research Unit and Department of Clinical and Experimental Medicine, University "Federico II," Naples; and Department of Lucania, San Carlo Hospital of Potenza, Potenza, and Madonna delle Grazie Hospital of Matera, Matera, Italy.

A. Cauli, MD, PhD, Consultant Rheumatologist, Department of Medical Science, Policlinico di Monserrato, University of Cagliari; E. Lubrano, $M D, P h D$, Consultant Rheumatologist, Rheumatology Unit, Department of Health Sciences, University of Molise; R. Scarpa, MD, Professor of Rheumatology, Research Unit and Department of Clinical and Experimental Medicine, University "Federico II"; I. Olivieri, MD, Director of Rheumatology, Department of Lucania, San Carlo Hospital of Potenza and Madonna delle Grazie Hospital of Matera.

Address correspondence to Prof. A. Cauli, Rheumatology Unit, Dipartimento di Scienze Mediche, Università di Cagliari, ss 554, Monserrato 09042, Italy 513157.E-mail cauli@medicina.unica.it in Naples, Italy, July 2011, a dedicated session on recent Italian contributions to PsA. Two speakers, Ignazio Olivieri (San Carlo Hospital, Potenza, and Madonna delle Grazie Hospital, Matera) and Ennio Lubrano (University of Molise, Campobasso) summarized these contributions in a session chaired by Raffaele Scarpa (University Federico II, Naples) and Alberto Cauli (University of Cagliari, Monserrato).

Dr. Olivieri underlined the importance of early diagnosis in $\mathrm{PsA}^{1}$ to avoid unnecessary examinations and avoid the risk of ineffective medication. The aim is to start effective treatment in the early phase of development and modify the natural course of the disease. Recent studies suggest that modern technology can identify entheseal and joint subclinical involvement in patients whose only evidence of disease is skin psoriasis (e.g., occult PsA) ${ }^{2}$. Dr. Olivieri also emphasized characterization of the clinical pattern of early PsA and presented results of a study performed on 47 patients with early-onset disease ( $\leq 12$ weeks' duration $)^{3}$, where previous treatment was only nonsteroidal antiinflammatory drugs (NSAID) to control joint symptoms. Clinical, articular ultrasonography, and total-body bone scintigraphy findings suggested that, in most cases, clinical oligo-enthesoarthritic presentation of early PsA might represent a polyarticular condition that is at increased risk for clinical progression. These findings influence the clinical decision-making process in patients with early PsA.

Dr. Olivieri next presented data suggesting a less satisfactory performance of the ClASsification for Psoriatic ARthritis criteria (CASPAR) when applied in early PsA ${ }^{4}$. However, the lower sensitivity of these criteria in this single-center cohort of patients was attributed to the small proportion of patients $(n=2)$ fulfilling the radiologic crite-

Personal non-commercial use only. The Journal of Rheumatology Copyright @ 2012 . All rights reserved. 
rion. This finding was subsequently tested in a 2-year prospective multicenter case-control study (unpublished observations), where the CASPAR criteria were applied to patients with early arthritis (disease duration $<2$ yrs) admitted to 8 Italian outpatient clinics. In this first prospective study of the CASPAR criteria in patients with early PsA, results demonstrated a high sensitivity $(91 \%)$ and specificity (97\%). The authors concluded that these criteria could be used for patients with early PsA in clinical trials and epidemiological studies.

The CASPAR criteria were also applied in a 24-week open-label trial at 5 Italian centers, where the effectiveness and safety of tumor necrosis factor- $\alpha$ (TNF- $\alpha$ ) blockers were assessed in a group of patients with early PsA $(n=29)$ with unsatisfactory responses to previous disease-modifying antirheumatic drugs ${ }^{5}$. The data demonstrated that TNF- $\alpha$ blockers improved the Disease Activity Score (DAS28) at 12 and 24 weeks compared with baseline; secondary clinical endpoints were also improved. No severe side effects were reported during the study, and no discontinuations from the study were reported. This study suggests that the TNF- $\alpha$ blockers are also effective in the treatment of early PsA.

Dr. Lubrano reported the results of several projects where radiological assessments of axial PsA were performed at Italian centers. In one study, the validity of the Bath AS Radiology Index (BASRI) and the modified Stoke AS Scoring System (mSASSS) scores were used to assess the radiological axial involvement in $\mathrm{PsA}^{6}$. Secondary endpoints included clinical, functional, and radiographic characteristics of axial involvement. Patients $(n=77)$ were enrolled if they fulfilled the CASPAR criteria and had clinical, functional, and/or radiological axial involvement. Three observers scored the radiographs by BASRI and mSASSS indexes. The construct validity was assessed by examining the correlation of instruments with patient-reported outcomes and anthropometric measures. Test-retest showed good reliability of both scores, but radiographs were more quickly read and scored with the BASRI index. The authors concluded that both the BASRI and the mSASSS are valid instruments for assessing spondylitis associated with PsA, but that longitudinal data are required to provide sensitivity to change of the scores.

Dr. Lubrano then discussed the development and validation of a modified index, the PsA Spondylitis Radiology Index (PASRI), for assessing the radiologic axial involvement in PsA in a multicenter cohort of patients with established disease ${ }^{7}$. Patients $(n=73)$ were enrolled based on clinical and/or radiologic criteria. The PASRI combined features of existing radiologic indices for AS with the addition of scores for the facet joints of the cervical and lumbar spine. Scores for the BASRI, the mSASSS, and the PASRI were obtained from standard radiographics. The construct validity of the PASRI was compared with patient-reported outcomes, e.g., the RLDQ (Revised Leeds Disability
Questionnaire) and BASFI (Bath AS Functional Index), and anthropometric measures. Data demonstrated that the PASRI presents a greater range in scoring the spinal radiologic features of PsA, provides fewer zero scores, and correlates well with anthropometric and patient-reported measures.

Finally, Dr. Lubrano reported the results of a multicenter observational study that investigated the effectiveness of the TNF- $\alpha$ antagonist etanercept on axial manifestations in 32 patients with established $\mathrm{PsA}^{8}$. Inclusion criteria were refractory PsA with axial manifestations and suitability for anti-TNF- $\alpha$ therapy. Effectiveness was defined according to the Assessment in AS (ASAS) response criteria (Bath AS Disease Activity Index: 50\% relative or absolute change of $20 \mathrm{~mm}$ and expert opinion in favor of continuation), and on the improvements of BASFI, anthropometric measures, Psoriasis Area and Severity Index (PASI), erythrocyte sedimentation rate, and C-reactive protein after 12 months of therapy. Data demonstrated that etanercept is effective on axial manifestations of established PsA, confirming the positive effects of anti-TNF- $\alpha$ therapy on clinical manifestations of the disease.

Several other contributions from the collaborative work of Italian centers have recently been published. One noteworthy example is PACE (PsA cost evaluation), a cost-of-illness study using TNF inhibitors in PsA patients with inadequate response to conventional therapy ${ }^{9}$. One hundred seven patients from 9 rheumatology centers and with different forms of PsA were given anti-TNF treatment, primarily etanercept. Information on resource use, health-related quality of life, disease activity, function, and laboratory data were collected at baseline and during the 12 months of therapy. Costs expressed in euros and utility as measured by EuroQol were compared before and after anti-TNF therapy to estimate the incremental cost per quality-adjusted life-year (QALY) gained; and the cost-effectiveness acceptability curve was calculated. The data showed a significant increase in direct cost due to the higher price of TNF inhibitors, which was only partially balanced by the decrease in indirect cost. However, a gain of 0.12 QALY resulted in a cost per QALY gained of $€ 40,876$ for the National Health Service (NHS) and of $€ 37,591$ for the taxpayers. The acceptability curve showed a $97 \%$ likelihood that anti-TNF therapy would be considered cost-effective at the (commonly accepted) willingness-to-pay threshold of $€ 60,000$ per QALY gained.

Of note, Italian centers were the largest contributors to a recent 319 patient GRAPPA and OMERACT (Outcome Measures in RA Clinical Trials) study on patient global assessment $(\mathrm{PGA})^{10}$. This study was devised to validate an instrument to evaluate patient perception of disease in randomized controlled trials as well as in daily clinical assessment in PsA. The study assessed the reliability of the PGA measured with a 0-100 $\mathrm{mm}$ visual analog scale (VAS), and

Personal non-commercial use only. The Journal of Rheumatology Copyright $@$ 2012. All rights reserved. 
the additional utility of separate VAS scales for joint and skin involvement only. Study data demonstrated the reliability of the instrument as evidenced by scores that were dependent on joints and skin but were not influenced by patient anxiety or depression. Joint and skin diseases were not found to correlate in terms of disease activity as evidenced by the swollen joint count versus PASI and by the joint versus skin VAS scores (both not significant). PGA assessed by means of VAS is therefore considered a reliable tool related to joint and skin disease activity. Because joint and skin diseases often diverge in patients with PsA, the authors suggested that in some circumstances both joint and skin scores should also be assessed.

Antonio Marchesoni led another collaborative Italian study to evaluate the clinical features associated with PsA and fibromyalgia $(\mathrm{FM})^{11}$. This multicenter cross-sectional study enrolled 266 patients with PsA and 120 patients with FM at 10 Italian rheumatologic centers. Standard clinical and laboratory data for PsA and FM were collected, as well as the presence of FM-related symptoms, response to NSAID, self-evaluation of pain, general health, disability, and the Fibromyalgia Impact Questionnaire. Analyses were focused on the clinical features shared by both conditions. The data demonstrated that FM patients had higher mean values of tender points, enthesitis score, and FM-related symptoms, and a lesser response to NSAID. In the multivariate analysis, the highest predictive values for FM were the presence of $\geq 6 \mathrm{FM}$-associated symptoms, $\geq 8$ more tender points, and no response to NSAID, all of which, the authors concluded, were the shared clinical features with the highest discriminating probability between PsA and FM.

A network of Italian centers with a common interest in PsA is now looking forward to new projects. One in particular is an 11 unit consortium led by Carlo Salvarani that will use a genome-wide scan to compare a cohort of patients with PsA and a matched cohort of patients with psoriasis alone with healthy control subjects. The objective of this study is to identify new genomic regions associated with genetic susceptibility and clinical expression of PsA, in the attempt to further our knowledge of the pathogenesis of PsA and define new treatment targets.

\section{REFERENCES}

1. Olivieri I, D'Angelo S, Padula A, Palazzi C. The challenge of early diagnosis of psoriatic arthritis. J Rheumatol 2008;35:3-5.

2. Palazzi C, Lubrano E, D'Angelo S, Olivieri I. Beyond early diagnosis: Occult psoriatic arthritis. J Rheumatol 2010;37:1556-8.

3. Scarpa R, Cuocolo A, Peluso R, Atteno M, Gisonni P, Iervolino S, et al. Early psoriatic arthritis: The clinical spectrum. J Rheumatol 2008;35:137-41.

4. D'Angelo S, Mennillo GA, Cutro MS, Leccese P, Nigro A, Padula A, et al. Sensitivity of the classification of psoriatic arthritis criteria in early psoriatic arthritis. J Rheumatol 2009;36:368-70.

5. Scarpa R, Atteno M, Lubrano E, Provenzano G, D’Angelo S, Spadaro A, et al. The effectiveness and safety of TNF-alpha blockers in the treatment of early psoriatic arthritis: An Italian multicentre longitudinal observational pilot study. Clin Rheumatol 2011;30:1063-7.

6. Lubrano E, Marchesoni A, Olivieri I, D'Angelo S, Spadaro A, Parsons WJ, et al. The radiological assessment of axial involvement in psoriatic arthritis: A validation study of the BASRI total and the modified SASSS scoring methods. Clin Exp Rheumatol 2009;27:977-80.

7. Lubrano E, Marchesoni A, Olivieri I, D’Angelo S, Spadaro A, Parsons WJ, et al. Psoriatic arthritis spondylitis radiology index: A modified index for radiologic assessment of axial involvement in psoriatic arthritis. J Rheumatol 2009;36:1006-11.

8. Lubrano E, Spadaro A, Marchesoni A, Olivieri I, Scarpa R, D'Angelo S, et al. The effectiveness of a biologic agent on axial manifestations of psoriatic arthritis. A twelve months observational study in a group of patients treated with etanercept. Clin Exp Rheumatol 2011;29:80-4.

9. Olivieri I, de Portu S, Salvarani C, Cauli A, Lubrano E, Spadaro A, et al. The psoriatic arthritis cost evaluation study: A cost-of-illness study on tumour necrosis factor inhibitors in psoriatic arthritis patients with inadequate response to conventional therapy. Rheumatology 2008;47:1664-70.

10. Cauli A, Gladman DD, Mathieu A, Olivieri I, Porru G, Tak PP, et al. Patient global assessment in psoriatic arthritis: A multicenter GRAPPA and OMERACT study. J Rheumatol 2011;38:898-903.

11. Marchesoni A, Atzeni F, Spadaro A, Lubrano E, Provenzano G, Cauli A, et al. Identification of the clinical features distinguishing psoriatic arthritis and fibromyalgia. J Rheumatol 2012;39:849-55. 